\title{
GRAU DE ADESÃO DE IDOSOS INTERNADOS EM UM HOSPITAL AO REGIME TERAPÊUTICO PARA HIPERTENSÃO E DIABETES
}

\author{
DEGREE OF ADHERENCE OF ELDERLY PATIENTS ADMITTED TO A HOSPITAL \\ TREATMENT REGIMEN FOR HYPERTENSION AND DIABETES
}

\section{Alyne Silva Britto ${ }^{a^{*}}$, Hellen Cristina de Almeida Abreu ${ }^{b^{*}}$, Elizete Aparecida Rubira ${ }^{c^{*}}$, Samira Reschetti Marcon ${ }^{\mathrm{d}^{*}}$, José Roberto Temponi de Oliveira ${ }^{*}$}

aalyne.britto@gmail.com, bafa_nany@yahoo.com.br, cearubira@gmail.com, dsamira.marcon@gmail.com, etemponi30@gmail.com *Universidade Federal de Mato Grosso - Cuiabá (MT), Brasil

\section{RESUMO}

Introdução: Os idosos convivem frequentemente com doenças crônico-degenerativas que podem ser prevenidas e controladas por regimes terapêuticos desenvolvidos pela medicina, sendo a eficácia, basicamente, dependente da adesão a eles. Objetivo: Avaliar o grau de adesáo dos idosos ao regime terapêutico para hipertensão e diabetes. Método: Pesquisa quantitativa com delineamento transversal. Foram entrevistados 30 idosos internados em uma instituição de saúde pública de Cuiabá (MT). Utilizou-se de questionário próprio com informaçôes relativas à identificação dos sujeitos, dados de saúde e doença autorreferidos e relativos à terapêutica, ao grau de adesão ao tratamento medicamentoso (teste de Morisky) e sobre o seguimento de orientaçóes de medidas náo farmacológicas. O período de coleta foi de julho de 2011 a janeiro de 2012. Resultados: 80,0\% dos idosos internados apresentaram baixo grau de adesão ao tratamento medicamentoso e 86,7\% nem sempre seguem as orientaçóes não farmacológicas (dieta apropriada, prática de atividade física regularmente, diminuir a ingestáo de sódio na dieta e reduzir o consumo bebida alcoólica). Conclusão: A elevada prevalência de hipertensão e diabetes em idosos e as devastadoras sequelas e consequências atribuídas ao inadequado controle destas condiçôes crônicas justificam a prioridade que deve ser dada à estratégia terapêutica, sendo de vital importância a identificação dos fatores relacionados à não aderência para que se possa obter resultados mais satisfatórios dos regimes terapêuticos prescritos.

Palavras-chave: Idoso; hipertensão; diabetes mellitus; doença crônica.

\section{ABSTRACT}

Introduction: The elderly often live with chronic diseases, which can be prevented and controlled by treatment regimens developed by medicine. And the effectiveness depends basically adequate adherence to these. Objective: To assess the degree of adherence to the therapeutic regimen for elderly hypertension and diabetes. Method: quantitative research with cross-sectional design. 30 elderly inpatients in a public health institution of Cuiabá - MT were interviewed. We used our own questionnaire with information identifying the subjects, and self-reported health data related to disease and treatment, the degree of adherence to medication (Morisky Test) and the following guidelines for non-pharmacological measures. The collection period was from July 2011 to January 2012 . Results: $80.0 \%$ of elderly patients admitted had a low degree of adherence to drug therapy and $86.7 \%$ do not always follow nonpharmacological guidelines (proper diet, regular practice of physical activity, decreasing sodium intake in the diet and reducing consumption liquor). Conclusion: The high prevalence of hypertension and diabetes in the elderly and the devastating sequelae and consequences attributed to inadequate control of these chronic 
conditions, justify the priority that should be given to the therapeutic strategy, so vital, and the identification of factors associated with noncompliance, so you can get more satisfactory results in the prescribed regimens

Keywords: Aged; hypertension; diabetes mellitus; chronic disease.

\section{Introdução}

A sociedade contemporânea passa por uma transição demográfica, evidenciada por um crescimento da população idosa. O envelhecimento do homem é um processo dinâmico e progressivo, com alteraçôes morfológicas, fisiológicas e psicológicas. Isso tem acarretado um aumento da prevalência de doenças crônicas, que necessitam de tratamentos complexos e de longa duração. Dentre estas, destacamos as doenças cardiovasculares $(\mathrm{DCV})$, que respondem pelo maior índice dos óbitos no país, elevando as despesas com assistência hospitalar no SUS e totalizando por volta de $75 \%$ dos gastos com atenção à saúde ${ }^{1,2}$.

Dentre os principais fatores de risco para DCV, a hipertensão arterial (HA) e o diabetes mellitus (DM) configuram importantes problemas, sendo as principais causas de morbimortalidade na população brasileira. Ambas são condiçôes crônicas que possuem elevadas taxas de prevalências, acarretando complicações agudas e crônicas. Frequentemente acometem mais os idosos, lembrando que a incidência aumenta com a idade ${ }^{3,4,5,6}$.

A prevalência de $\mathrm{HA}$ em pacientes diabéticos é pelo menos duas vezes a da população em geral ${ }^{2,3,7}$. Nesse sentido, diversos estudos sobre HA e DM têm mostrado que o controle rigoroso da glicemia e da pressão arterial é capaz de reduzir as complicaçôes e os tratamentos dos problemas crônicos de saúde tem como finalidade reduzir a morbimortalidade e manter a qualidade de vida das pessoas enfermas ${ }^{3,4,5,8}$.

Para tal, na HA utilizam-se medidas não medicamentosas isoladas ou associadas a medicamentos anti-hipertensivos. Várias abordagens no tratamento resultam na redução dos níveis de pressão arterial e provocam também alteraçóes na qualidade de vida do pacientes.

Já para a terapêutica do DM, recomenda-se, inicialmente, o uso de medidas não farmacológicas. Se as medidas iniciais não forem suficientes, indica-se o uso de medicamentos, monoterapia oral ou combinação oral com medicamentos que tenham açóes farmacológicas diferentes. Quando não se obtém o controle, inicia-se insulinoterapia isolada ou associada à medicação oral. As medidas não farmacológicas incluem mudanças nos hábitos alimentares, através de orientação médica ou de nutricionistas, e atividade física orientada ${ }^{10,11}$.

A modificação no estilo de vida, como é denominada a terapia não farmacológica, inclui a redução do peso corporal, da ingestão do sal e gorduras, do consumo de bebidas alcoólicas, o fim do tabagismo e a prática de exercícios físicos com regularidade. Elas são indicadas tanto para um tratamento inicial quanto para associação com a terapia farmacológica. De baixo custo e risco mínimo, atuam no aumento da eficácia do tratamento medicamentoso e no controle de outros fatores de risco cardiovasculares ${ }^{1}$.

Assim, vários fatores podem influenciar a adesão ao tratamento, tais como idade, gênero, etnia, estado civil, escolaridade, nível socioeconômico, autoestima, crenças de saúde, hábitos de vida e culturais, cronicidade da doença, ausência de sintomas e consequências tardias decorrentes da doença, custo do tratamento, efeitos indesejáveis do tratamento, esquemas terapêuticos complexos, o acesso ao serviço de saúde e às políticas de saúde, e, finalmente, o relacionamento com a equipe de saúde ${ }^{9,12}$.

O desenvolvimento de pesquisas que buscam entender os aspectos que levam o idoso a não adesão (ou adesão inadequada) do tratamento pode contribuir para a elaboraçáo de intervençóes mais eficazes em educação em saúde para esse grupo etário, contribuindo para a redução dos altos índices de internação, morbidade e mortalidade, além de melhorar a qualidade de vida deles. A partir disso, este estudo tem como objetivo avaliar o grau de adesão ao regime terapêutico (farmacológico e não farmacológico) de idosos com hipertensão e/ou diabetes internados em um hospital universitário.

\section{Método}

Trata-se de um estudo descritivo de corte transversal, realizado em um hospital de ensino e público que presta serviços de baixa, média e alta complexidade, referência na cidade de Cuiabá, no Estado de Mato Grosso.

Participaram do estudo 30 idosos com idade igual ou superior a 60 anos de ambos os sexos, com diagnóstico de HA e/ou DM, internados no período de julho de 2011 a janeiro de 2012 nas clínicas médica e cirúrgica. Foram considerados como critérios de inclusão deste estudo: ter 60 anos ou mais; ter o diagnóstico de $\mathrm{HA}$ e/ou de DM; estar internado na instituição, não apresentar confusão mental, compreender e concordar com os objetivos e metodologia do estudo e assinar o TCLE. 
A aplicação do questionário foi realizada diretamente com o paciente, na própria clínica. Os dados foram coletados por meio de um questionário próprio, que teve como referência para elaboração, a Caderneta de Saúde da Pessoa Idosa $^{13}$ e o formulário utilizado para o cadastramento de hipertensos e diabéticos no programa Hiperdia ${ }^{14}$.

Utilizou-se um questionário que contemplava informaçóes sócio-demográficas (sexo, idade, grau de escolaridade, renda familiar, com quem mora, estado civil e ocupação) e dados sobre as condiçôes de saúde (histórico familiar de HA e DM, diagnóstico, tempo de diagnóstico, uso de bebida alcoólica, uso de tabaco, prática de atividade física, complicaçóes referentes à HA e DM, internaçóes, procura às unidades de saúde para verificar pressão arterial e/ou glicemia e realizar automedida em sua residência da pressão arterial e/ ou glicemia). Além disso, dados relativos à terapêutica, como o tipo de tratamento, medicamentos que utilizava, quantos comprimidos utilizava diariamente e orientaçóes de medidas não farmacológicas (dieta apropriada, prática de atividade física, diminuição da ingestão de sal e de bebida alcoólica) e o grau de adesão ao tratamento segundo o teste de Morisky ${ }^{15}$.

Para identificar o grau de adesão ao tratamento medicamentoso, utilizou-se o teste de Morisky ${ }^{13}$, que é composto por quatro perguntas que buscam avaliar o comportamento do paciente em relação ao uso habitual do medicamento. Considera-se alto grau de adesão quando todas as respostas são negativas. Porém, quando pelo menos uma das respostas é afirmativa, o paciente é classificado no grupo de baixo grau de adesão.

Os dados foram digitados e analisados no programa SPSS 14.0, onde foram realizadas análises descritivas e inferências para verificar a correlação entre as variáveis.

Este estudo foi submetido à apreciação e teve a aprovaçáo do comitê de ética e pesquisa do Hospital Universitário Julio Muller, parecer No 63/2011. Todos os sujeitos que concordaram em participar da pesquisa fizeram a leitura do Termo de Consentimento Livre e Esclarecido (TCLE) e, subsequente, a assinatura do mesmo.

\section{Resultados}

As características demográficas e sociais dos 30 idosos incluídos no estudo, hipertensos e/ou diabéticos, internados na clínica médica e clínica cirúrgica, são apresentadas na Tabela 1 . Houve predomínio do sexo masculino $19(63,3 \%), 46,7 \%(n=21)$ com faixa etária entre 65 e 75 anos, 50,0\% ( $n=15)$ casados e com baixo grau de escolaridade $(\mathrm{N}=11 ; 36,7 \%)$ com 1 a 4 anos de estudo. Quanto à renda, 73,3\% $(n=22)$ recebem um salário mínimo e $70 \%(\mathrm{n}=21)$ eram aposentados.
Tabela 1: Distribuição dos idosos dos idosos hipertensos e/ou diabéticos internados na clínica médica e cirúrgica segundo características sóciodemográficas. Cuiabá (MT), 2012

\begin{tabular}{|c|c|c|}
\hline Variáveis & n & $\%$ \\
\hline \multicolumn{3}{|l|}{ Sexo } \\
\hline Feminino & 11 & 36,7 \\
\hline Masculino & 19 & 63,3 \\
\hline \multicolumn{3}{|l|}{ Idade } \\
\hline 60 a 64 anos & 9 & 30,0 \\
\hline 65 a 75 anos & 14 & 46,7 \\
\hline 76 anos ou mais & 7 & 23,3 \\
\hline \multicolumn{3}{|l|}{ Estado civil } \\
\hline Casado & 15 & 50,0 \\
\hline Solteiro & 4 & 13,3 \\
\hline Viúvo & 9 & 30,0 \\
\hline Separado & 2 & 6,7 \\
\hline \multicolumn{3}{|l|}{ Grau de } \\
\hline \multicolumn{3}{|l|}{ escolaridade } \\
\hline Analfabeto & 10 & 33,3 \\
\hline 1 a 4 anos & 11 & 36,7 \\
\hline 5 a 8 anos & 4 & 13,3 \\
\hline Mais de 8 anos & 5 & 16,7 \\
\hline \multicolumn{3}{|l|}{ Ocupação } \\
\hline Aposentado & 21 & 70,0 \\
\hline Autônomo & 4 & 13,3 \\
\hline $\begin{array}{l}\text { Empregado com } \\
\text { carteira assinada }\end{array}$ & 2 & 6,7 \\
\hline Do lar & 3 & 10,0 \\
\hline \multicolumn{3}{|l|}{ Renda familiar } \\
\hline Sem renda & 2 & 6,7 \\
\hline 1 salário mínimo & 22 & 73,3 \\
\hline $\begin{array}{l}2 \text { a } 4 \text { salários } \\
\text { mínimos }\end{array}$ & 6 & 20,0 \\
\hline Total & 30 & 100,0 \\
\hline
\end{tabular}

A Tabela 2 apresenta os dados de saúde e doença dos idosos, que revela que $21(70,0 \%)$ são hipertensos e 7 $(23,3 \%)$ hipertensos e diabéticos. A maioria afirmou saber do diagnóstico por mais de seis anos, e dos idosos diabéticos $(n=20 ; 66,7 \%)$. Em relação às complicaçôes dessas morbidades, 16,7\% ( $n=5)$ foram acometidos por Acidente Vascular Encefálico (AVE). No que se refere às características do tratamento, houve predomínio do tratamento medicamentoso ( $\mathrm{n}=27 ; 90,0 \%)$. E a quantidade de comprimidos ingeridos pelos idosos diariamente para o controle da pressão arterial e dos níveis de glicemia foi de 40,0\% ( $n=12)$ quatro ou mais de seis comprimidos.

Quanto ao grau de adesão ao tratamento medicamentoso, segundo o teste de Morisky, observou que: 24 $(82,7 \%)$ apresentaram baixo grau de adesão ao tratamento medicamentoso e somente $5(17,2 \%)$ apresentaram alto grau de adesão ao tratamento medicamentoso, conforme a Tabela 3 . 
Tabela 2: Distribuição dos idosos dos idosos hipertensos e/ou diabéticos internados na clínica médica e cirúrgica segundo condições de saúde. Cuiabá (MT), 2012

\begin{tabular}{|c|c|c|}
\hline Variáveis & $\mathbf{n}$ & $\%$ \\
\hline \multicolumn{3}{|l|}{ Patologia } \\
\hline Hipertensão arterial & 21 & 70,0 \\
\hline Diabetes mellitus I & 1 & 3,3 \\
\hline $\begin{array}{l}\text { Diabetes } \\
\text { mellitus II }\end{array}$ & 1 & 3,3 \\
\hline $\begin{array}{l}\text { Hipertensão arterial } \\
\text { e diabetes mellitus }\end{array}$ & 7 & 23,3 \\
\hline \multicolumn{3}{|l|}{ Histórico familiar } \\
\hline Hipertensão arterial & 14 & 46,7 \\
\hline Diabetes mellitus & 3 & 10,0 \\
\hline \multicolumn{3}{|l|}{ Tempo de diagnóstico } \\
\hline \multicolumn{3}{|l|}{ Hipertensão arterial } \\
\hline $\begin{array}{l}\text { Menos de } 1 \text { ano e } \\
11 \text { meses }\end{array}$ & 4 & 13,3 \\
\hline $\begin{array}{l}\text { de } 4 \text { a } 5 \text { anos e } 11 \\
\text { meses }\end{array}$ & 2 & 6,7 \\
\hline Mais de 6 anos & 20 & 66,7 \\
\hline \multicolumn{3}{|l|}{ Diabetes mellitus } \\
\hline $\begin{array}{l}4 \text { a } 5 \text { anos e } 11 \\
\text { meses }\end{array}$ & 5 & 16,7 \\
\hline Mais de 6 anos & 3 & 10,0 \\
\hline \multicolumn{3}{|l|}{ Complicaçóes } \\
\hline $\begin{array}{l}\text { Acidente Vascular } \\
\text { Encefálico (AVE) }\end{array}$ & 5 & 16,7 \\
\hline $\begin{array}{l}\text { Infarto Agudo do } \\
\text { Miocárdio (IAM) }\end{array}$ & 2 & 6,6 \\
\hline AVE e IAM & 2 & 6,7 \\
\hline \multicolumn{3}{|l|}{ Tratamento } \\
\hline Medicamentoso & 27 & 90,0 \\
\hline $\begin{array}{l}\text { Medicamentoso e } \\
\text { fitoterápico }\end{array}$ & 2 & 6,7 \\
\hline Não medicamentoso & 1 & 3,3 \\
\hline \multicolumn{3}{|c|}{ Quantos comprimidos diariamente? } \\
\hline Apenas 1 & 4 & 13,3 \\
\hline 2 a 3 & 8 & 26,7 \\
\hline 4 a 5 & 8 & 26,7 \\
\hline 6 ou mais & 10 & 33,3 \\
\hline Total & 30 & 100,0 \\
\hline
\end{tabular}

Tabela 3: Distribuição dos idosos dos idosos hipertensos e/ou diabéticos internados na clínica médica e cirúrgica segundo o grau de adesão ao tratamento medicamentoso. Cuiabá (MT), 2012

\begin{tabular}{lcc}
\hline Variáveis & $\mathbf{N}$ & $\%$ \\
Alto grau de adesão & 5 & 17,2 \\
Baixo grau de adesão $^{*}$ & 24 & 82,7 \\
Total $^{*}$ & 29 & 100,0 \\
\hline $\mathrm{N}=29$, pois um idoso não realiza tratamento medicamentoso
\end{tabular}

Quanto ao cumprimento das orientaçóes de medidas não farmacológicas dadas pela equipe de saúde, observou-se que $23(76,7 \%)$ dos idosos fazem dieta apropriada; $21(70,0 \%)$ não praticam nenhuma atividade física; 18 (60\%) seguem a orientação de baixa ingestão de sódio na dieta, e quanto à redução do consumo de bebida alcoólica, 22 (73,3\%) dos idosos seguem esta orientação. Observamos também que 26 (86,7\%) dos idosos deste estudo nem sempre seguem as orientaçóes não farmacológicas e apenas 4 (13,3\%) afirmam que sempre seguem as orientaçóes não farmacológicas, conforme apresentados na Tabela 4.

Tabela 4: Distribuição dos idosos dos idosos hipertensos e/ou diabéticos internados na clínica médica e cirúrgica segundo medidas não farmacológicas. Cuiabá (MT), 2012

\begin{tabular}{lcc}
\hline Variáveis & n & $\%$ \\
$\begin{array}{l}\text { Orientaçáo de medidas não } \\
\text { farmacológicas }\end{array}$ & & \\
$\begin{array}{l}\text { Sempre seguem as orientações } \\
\text { não farmacológicas }\end{array}$ & 4 & 13,3 \\
$\begin{array}{l}\text { Nem sempre seguem as orientações } \\
\text { não farmacológicas } \\
\text { Total }\end{array}$ & 26 & 86,7 \\
\hline
\end{tabular}

\section{Discussão}

Neste estudo, observou-se a prevalência de idosos portadores de HA e DM do gênero masculino, com a faixa etária entre 65 e 75 anos. Provavelmente, a maioria da populaçáo é formada por homens pelo fato de serem a maioria entre os idosos internados ${ }^{16}$. Além disso, indicadores demográficos populacionais, relacionados ao gênero, apontam que os idosos do sexo masculino apresentam maiores riscos de adoecer e morrer quando comparados aos do sexo feminino ${ }^{17}$.

Quanto à escolaridade, houve predomínio da baixa escolaridade. O baixo nível de escolaridade pode ser consequência do pouco acesso à escola quando crianças. Esse dado apresenta-se como um desafio para os profissionais, que devem abordar as questóes de saúde e as intervençóes de uma maneira compreensível, de acordo com as necessidades de cada indivíduo ${ }^{17}$.

Quanto ao tipo de morbidade, observou-se que mais da metade dos idosos eram hipertensos. Isso acontece pelo fato da HA variar de 50 a $70 \%$ na população geriátrica, aumentando linearmente com a idade $^{9}$. Nos diabéticos a hipertensão arterial é duas vezes mais frequente do que na população em geral ${ }^{18}$. Esta situação é incrementada pela ausência de atividade física e dieta inadequada, tornando um grave fator de risco para ocorrências de doenças cardiovasculares ${ }^{19}$.

Para tanto, a abordagem da hipertensão arterial e da diabetes é constituída de intervenção farmacológica e não farmacológica sempre acompanhadas por mudanças 
no estilo de vida. Neste sentido, o sucesso do controle das doenças crônicas depende, sobretudo, da adesão adequada do paciente ao tratamento e às práticas de saúde que estimulem ou facilitem a mudança do estilo de vida ${ }^{4}$.

Assim, a adesão ao tratamento em pacientes crônicos representa a extensão em que o comportamento da pessoa coincide com o aconselhamento dado pelo profissional de saúde. Trata-se de um processo dinâmico, influenciado por vários fatores, que requer um acompanhamento cuidadoso, com intervençôes específicas em termos individuais em que a família, as organizaçóes e a comunidade em geral têm um papel importantíssimo ${ }^{20}$.

Nossos achados por meio do teste de Morisky mostraram que mais da metade dos idosos com hipertensão arterial e/ou diabetes apresentavam comportamento indicativo de baixo grau de adesão, sendo os idosos considerados muito vulneráveis quanto a adesão ${ }^{21}$. Os resultados corroboram com um estudo realizado com idosos hipertensos atendidos em um centro de saúde de Novo Horizonte, São Paulo, Brasil, que avaliou a adesão pelo teste de Morisky, onde apenas 28\% dos indivíduos tiveram adesão ao tratamento ${ }^{22}$. Entretanto, uma pesquisa realizada em Santa Catarina em 2012, que avaliou a adesão aos medicamentos em idosos hipertensos pelo TMG, apresentou maior prevalência de adesão ao tratamento ${ }^{23}$.

A baixa adesão é complexa e multifatorial, associado a distintos fatores, como os relacionados ao doente, os sociodemográficos, os culturais e os comportamentais, os relacionados aos medicamentos e aos vários aspectos clínicos, os ambientais e os econômicos ${ }^{24}$.

Neste contexto, temos que ressaltar o fator relacionado ao uso de medicaçóes, visto que, os estudos apontam que $80 \%$ dos idosos fazem uso de algum medicamento. Assim, o uso racional destes, trouxe muitos ganhos para a saúde nas últimas décadas, como os medicamentos que controlam a hipertensão arterial e o diabetes, que contribuem para a prevenção de Acidentes Vasculares Cerebrais e Infarto do Miocárdio e, consequentemente, evitam muitas mortes prematuras. No entanto, a utilização dos fármacos requer cuidados específicos para evitar interaçóes, reações adversas, controlar efeitos secundários para aumentar a qualidade de vida e acarretar em uma diminuição da morbilidade e da mortalidade ${ }^{21}$.

Quanto a aderir medidas não farmacológicas, a maioria dos idosos confirmou que nem sempre segue as orientações. Em um estudo realizado no hospital universitário do Rio Grande do Sul observou-se também que poucos idosos eram aderentes ao tratamento não farmacológico $(18 \%)^{25}$. Assim, as recomendaçóes de mudanças no estilo de vida relacionadas ao tratamento não farmacológico apresentam geralmente percentuais mais elevados de não adesão quando comparadas ao tratamento farmacológico, apesar de diversas pesquisas comprovarem que a "adesáo" a essas orientaçóes podem impactar significativamente os sintomas, a capacidade funcional, o bem-estar, a morbidade e o prognóstico da doença ${ }^{21}$.

A adesão terapêutica requer uma abordagem multidisciplinar com intervençóes concentradas em todos os intervenientes no processo de cuidar. As estratégias de intervenção têm como objetivo melhorar a adesão dos doentes ou promover o desenvolvimento de competências dos profissionais para aumentar a adesão de quem cuidam. Essas intervenções podem ser classificadas em educacionais e comportamentais. As educacionais incluem o fornecimento de informação escrita ou oral e material audiovisual (pelo médico, enfermeiro, farmacêutico, dentre outros) individualmente ou em grupo. Já as intervençóes comportamentais contribuem para aumentar a comunicação e aconselhamento, simplificar os regimes terapêuticos. Apesar de essas intervençóes estarem identificadas, ainda não se conhece bem a sua eficácia ${ }^{3,7,21}$.

É grande a repercussão da não adesão a regimes terapêuticos entre idosos ${ }^{1}$, tornando esse um dos grandes problemas de saúde no mundo em face da elevada prevalência de doenças crônicas e sendo responsável pelo aumento da probabilidade do insucesso da terapêutica por complicaçóes evitáveis, por aumento dos gastos com a saúde, aumento da morbidade $e$ mortalidade ${ }^{26}$.

\section{Conclusão}

Foi alta a não adesão ao tratamento medicamentoso e não farmacológico dos idosos internados na clínica médica e cirúrgica. Neste estudo também foram analisados possíveis aspectos que afetam a adesão ao tratamento, como os sócio-demográficos e os relacionados à doença e à terapêutica. Porém, destaca-se a necessidade de avaliar, além desses fatores, os relacionados aos profissionais e ao sistema de saúde, gerando, assim, uma maior atenção para aspectos da atenção à saúde, como a comunicação, a preocupação no investimento na informação (educação em saúde), multidisciplinaridade e também a atualização dos profissionais de saúde. Faz-se necessária uma discussão entre equipe multidisciplinar de saúde, idosos, familiares e gestores sobre o problema da não adesão ao tratamento, bem como possibilidades para resolução, pois a não adesão compromete a qualidade de vida do idoso.

\section{Agradecimento}

Ao hospital, pelo auxílio na operacionalização deste estudo. 


\section{Referências}

1. Almeida HO, Versiani ER, Dias AR, Novaes MRCG, Trindade, EMV. Adesão a tratamentos entre idosos. Comun Ciênc Saúde. 2007;18(1):57-67.

2. Brasil. Ministério da Saúde. Secretaria de Atenção à Saúde. Departamento de Açôes Programáticas e Estratégicas. Atenção à saúde da pessoa idosa e envelhecimento/ Ministério da Saúde, Secretaria de Atenção à Saúde, Departamento de Ações Programáticas e Estratégicas, Área Técnica Saúde do Idoso. Brasília, 2010.

3. Santos PA, Pinho CPS. Diabetes mellitus em pacientes coronariopatas: prevalência e fatores de risco cardiovascular associado. Rev Bras Clin Med. 2012;10(6):469-75.

4. Silva TR, Feldmam C, Lima MHA, Nobre MRC, Domingues RZL. Controle de diabetes mellitus e hipertensão arterial com grupos de intervenção educacional e terapêutica em seguimento ambulatorial de uma Unidade Básica de Saúde. Saude Soc. 2006;15(3):180-189.

5. Monteiro LZ, Fiani CRV, Freitas MCF, Zanetti ML, Foss MC. Redução da pressão arterial, da IMC e da glicose após treinamento aeróbico em idosas com diabete tipo 2. Arq Bras Cardiol. 2010;95(5):563-570.

6. Martins LN, Souza LS, Silva CF, Machado RS; Silva CEF, Vilagra MC, et al. Prevalência dos fatores de risco cardiovascular em adultos admitidos na unidade de dor torácica em Vassouras, RJ. Rev Bras Cardiol. 2011;24(5):299-307.

7. Knuth AG, Bielemann RM, Silva SG, Borges TT, Del Duca GF, Kremer MM, et al. Conhecimento de adultos sobre o papel da atividade física na prevenção e tratamento de diabetes e hipertensão: estudo de base populacional no Sul do Brasil. Cad. Saúde Pública. 2009;25(3):513-520.

8. Reiners AAO, Azevedo RCS, Vieira MA, Arruda ALG. Produção bibliográfica sobre adesão/não adesão de pessoas ao tratamento de saúde. Ciênc Saúde Coletiva. 2008;13(Suppl 2):2299-2306.

9. Vitor RS, Sakai FK, Consoni, PRC. Indicação e adesão de medidas não farmacológicas no tratamento da hipertensão arterial. Rev AMRIGS. 2009;53(2):117-121.

10. Santos FS, Oliveira KR, Colet CF. Adesão ao tratamento medicamentoso pelos portadores de Diabetes Mellitus atendidos em uma Unidade Básica de Saúde no município de Ijuí/RS: um estudo exploratório. Rev Ciênc Farm Básica Apl. 2010;31(3):223-227.

11. Espírito Santo MB, Souza LME, Souza ACG, Ferreira FM, Silva CNMR, Taitson PF. Adesão dos portadores de diabetes mellitus ao tratamento farmacológico e não farmacológico na atenção primária à saúde. Enf Rev. 2012;15(1):88-101.

12. Rocha CH, Oliveira APS, Ferreira C, Faggiani FT, Schroeter G, de Souza ACA, et al. Adesão à prescrição médica em idosos de Porto Alegre, RS. Ciênc. Saúde Colet. 2008;13(Suppl):703-710.

13. Brasil. Ministério da Saúde. Caderneta de saúde da pessoa Idosa. Brasília: Ministério da Saúde; 2007.

14. Brasil, Ministério da Saúde. Plano de reorganização da atenção à hipertensão arterial e ao diabetes mellitus. Manual de hipertensão arterial e diabetes mellitus. Brasília: Ministério da Saúde; 2002.

15. Morisky DE, Green LW, Levine DM. Concurrent and predictive validity of a self-reported measure of medication adherence. Med Care. 1986;24(1):67-74.

16. Brasil. Ministério da Saúde. DATASUS. Morbidade Hospitalar do SUS. Disponível em: http://www2.datasus. gov.br/DATASUS/index.php? area=0205\&VObj=http:// tabnet.datasus.gov.br/cgi/ deftohtm.exe?sim/cnv/obt10. Acesso em julho de 2012.

17. Santos MIPO. Perfil dos idosos internados no Hospital Geral em Belém (Pará). Esc. Anna Nery. 2007;11(1):23-29.

18. Spinel LF, Püschel VAA. Perfil de estilo de vida de pessoas com doença cardiovascular. Rev Gaúcha Enferm. 2007;28(4):534-41.

19. Molina MCB, Cunha RS, Herkenhoff LF, Mill JG. Hipertensão arterial e consumo de sal em população urbana. Rev Saúde Pública. 2003;37(6):743-750.

20. Pereira JC, Barreto SM, Passos VMA. O perfil de saúde cardiovascular dos idosos brasileiros precisa melhorar: estudo de base populacional. Arq Bras Cardiol. 2008;91(1):1-10.

21. Henriques MAP. Adesão ao regime medicamentoso em idosos na comunidade: eficácia das intervençóes de enfermagem [tese]. Lisboa: Universidade de Lisboa; 2006.

22. Eid LP, Nogueira MS, Veiga EV, Cesarino EJ, Alves LMM. Adesão ao tratamento anti-hipertensivo: análise pelo Teste de Morisky-Green. Rev Eletr Enf. 2013;15(2):362-7

23. Pucci N, Pereira MR, Vinholes DB, Pucci P, Campos ND. Conhecimento sobre hipertensao arterial sistêmica e adesão ao tratamento anti-hipertensivo em idosos. Rev Bras Cardiol. 2012;25(4):322-9

24. Castro RA, Aliti GB, Linhares JC, Rabelo ER. Adesão ao tratamento de pacientes com insuficiência cardíaca em um hospital universitário. Rev Gaúcha Enferm. 2010;31(2):225-31.

25. Santos ZMSA, Frota MA, Cruz DM, Olanda SDO. Adesão do cliente hipertenso ao tratamento: análise com abordagem interdisciplinar. Texto Contexto Enferm. 2005 jul./ set;14 (3): 332-40.

26. Dewulf NLS, Monteiro RA, Passos ADC, Vieira EM, Troncon LEA. Adesão ao tratamento medicamentoso em pacientes com doenças gastrintestinais crônicas acompanhados no ambulatório de um hospital universitario. Rev Bras Ciênc Farm. 2006;42(4):575-584. 